Max-Planck-Institut für demografische Forschung Max Planck Institute for Demographic Research Konrad-Zuse-Strasse 1 - D-18057 Rostock - GERMANY

Tel +49 (0) 3812081 - 0; Fax +49 (0) 3812081 - 202;

http://www.demogr.mpg.de

MPIDR WORKING PAPER WP 2003-040

DECEMBER 2003

\title{
Lifesaving increases Life Expectancy
}

M.S. Finkelstein (FinkelM@sci.uovs.ac.za)

This working paper has been approved for release by: James W. Vaupel (jwv@ demogr.mpg.de)

Head of the Laboratory of Survival and Longevity.

(C) Copyright is held by the authors.

Working papers of the Max Planck Institute for Demographic Research receive only limited review. Views or opinions expressed in working papers are attributable to the authors and do not necessarily reflect those of the Institute. 


\title{
LIFESAVING INCREASES LIFE EXPECTANCY
}

\author{
M.S. Finkelstein \\ Department of Mathematical Statistics \\ University of the Free State \\ PO Box 339, 9300 Bloemfontein, Republic of South Africa \\ (e-mail: FinkelM@sci.uovs.ac.za) \\ and \\ Max Planck Institute for Demographic Research \\ Rostock, Germany
}

Draft 19 November 2003

\begin{abstract}
The notion of repeated minimal repair is analyzed and applied to modeling the lifesaving procedure of organisms. Under certain assumptions the equivalence between demographic lifesaving model and reliability shock model is proved. Both of these models are based on the non-homogeneous Poisson processes of underlying potentially harmful events The lifesaving ratio for homogeneous and heterogeneous populations is defined. Some generalizations are discussed. Several simple examples are considered.
\end{abstract}

Keywords: minimal repair, statistical minimal repair, information based minimal repair

\section{INTRODUCTION}

According to a number of authors (see Yashin et al. (2000) for references) the DNA repair capacity can be responsible for aging of humans. One can assume that in the absence of the proper repair spontaneous DNA mutation leads to death of an organism (Yashin et al. (2000)). In a more general evolutionary setting certain resources are allocated for the organism maintenance during the life cycle and failure to perform the maintenance action also leads to death. These theories show an importance of a natural repair (maintenance) mechanism for modeling the life span characteristics of organisms.

On the other hand, numerous advances in healthcare result in saving lives, where previously these lives were lost. It is clear that successful medical treatments can be also formally considered as some overall repairs of an organism. Therefore, the stochastic modeling of repair actions and of the corresponding impact on resulting mortality rates seems to be an important and interesting problem both from theoretical and practical points of view. Due to obvious analogies, it seems rather natural to use methods and approaches of stochastic theory of repairable engineering systems for the life span and mortality rate modeling of humans. It should be kept in mind, however, that these methods should be applied very carefully, taking into account a number of important specific features.

Denote by $F(t)$ the cumulative distribution function (Cdf) of a time to failure (death) of an object (organism) and by $f(t)$ and $\lambda(t)$ its probability density function (pdf) and the failure rate (mortality rate), respectively. Assume that the repair action is performed instantaneously upon failure. The repair is usually qualified as perfect, if the Cdf of the repaired object is as good as new: $F(t)$. Traditionally (see, e.g., Bar- 
low and Proschan (1975)) a minimal repair at time $x$ is defined by the following Cdf of time to failure of the restored object

$$
F(t \mid x) \equiv 1-\frac{1-F(t+x)}{1-F(x)} .
$$

Therefore, definition (1) means that the Cdf of the restored object is the same as the Cdf of the residual lifetime of statistically identical object, which had survived in $[0, x]$.

A convenient mathematical description of repair processes uses the concept of the hazard rate process (failure intensity) $\lambda_{t}, t \geq 0$ (Aven, Jensen (1999), Finkelstein (2003)). Repairs can be perfect, minimal or imperfect. (See e.g., Finkelstein (2002) for the definition and properties of imperfect repair.) The classical example of $\lambda_{t}$ is the hazard rate process defined by the renewal process: the perfect repair, when after each repair an object is 'as good as new'. Additional assumption stating that the failure rate $\lambda(t)$ of the underlying Cdf $F(t)$ is a monotone function, should be also imposed:

$$
\lambda_{t}=\sum_{n=0}^{\infty} \lambda\left(t-T_{n}\right) I\left(T_{n}<t \leq T_{n+1}\right), T_{0}=0,
$$

where $T_{n}$ is the instant of $n$th repair, $n \geq 0$.

Another standard example is the deterministic hazard rate process:

$$
\lambda_{t}=\lambda(t),
$$

which, in fact, defines the non-homogeneous Poisson (NHPP) process of repairs with intensity $\lambda(t)$. It is clear that in accordance with definition (1), the NHPP can be alternatively defined as the process of minimal repairs.

We shall study in this paper the lifesaving procedure, which restores an organism to the state it had just prior some harmful event that could cause death. Therefore, the model (3) seems to be reasonable in this respect and it is rather unrealistic that some repair action in the organism can bring it to 'as good as new state', as in relation (2). On the other hand, intermediate imperfect repair is worth considering as well, but there are certain mathematical hurdles on this way. We shall briefly discuss this problem in Section 4.

\section{REPEATED RESUSCITATION REVISITED}

Let $P_{t}, t \geq 0$ denotes the NHPP of harmful events with intensity $\lambda(t)$, which potentially can lead to death (diseases, disorders etc.). Another interpretation (Yashin et al (2000)) is that $\lambda(t)$ is the mortality rate in a given population without possibility of repair. If we assume the possibility of only $n$ minimal repairs, then the new (better) mortality rate is obviously given by the following relation (Vaupel and Yashin (1987)):

$$
\lambda_{n}(t)=\lambda(t) \frac{\Lambda^{n}(t)}{n !\left(1+\Lambda(t)+\frac{\Lambda^{2}(t)}{2}+\ldots+\frac{\Lambda^{n}(t)}{n !}\right)},
$$

where $\Lambda(t)=\int_{0}^{t} \lambda(x) d x$ is the cumulated mortality rate. This approach can be easily generalized to the case when the number of possible minimal repairs is random. 
Another and maybe more realistic generalization from the demographic point of view (Vaupel and Yashin (1987)) is as follows. Let $\lambda(t)$ be the mortality rate for a homogeneous population. Suppose that due to some reason (e.g., better healthcare) $\lambda(t)$ is reduced to a new level $\lambda_{r}(t)$ to be modeled by some function $\theta(t), 0<\theta(t) \leq 1, \forall t \geq 0$ :

$$
\lambda_{r}(t)=\theta(t) \lambda(t)
$$

The following lifesaving procedure gives a useful interpretation of equation (4): each life, characterized by initial mortality rate $\lambda(t)$ is saved (cured) with probability $1-\theta(t)$ (or equivalently a proportion of individuals who would have died are now resuscitated and given another chance). Those who are saved, experience the minimal repair. The number of resuscitations (repairs) is unlimited. Under these assumptions, it was proved analytically in Vaupel and Yashin (1987) that the described lifesaving procedure results in the mortality rate given by equation (4). As a corollary to this result, the point process of saved lives is also the NHPP with intensity

$$
\lambda_{s}(t)=(1-\theta(t)) \lambda(t) \text {. }
$$

The similar result was obtained for different reliability related settings by Brown and Proschan (1983) (for $\theta(t) \equiv \theta$ ), Block et al (1985), Finkelstein (1999). The following shock model (Finkelstein (1999)) gives another useful interpretation and, in fact, presents an elegant and more general non-technical proof.

Consider firstly an object (organism) subject to a general orderly stochastic point process of shocks (e.g., diseases). Assume that a shock, affecting an object at time $t \in(0, \infty)$, independently of the previous shocks, causes a failure (death) with probability $\theta(t)$ and does not cause any changes in the object with a complementary probability $1-\theta(t)$. Assume for simplicity that this is the only cause of failure of an object. Let $\lambda_{c}\left(t, H_{t}\right)$ denotes the corresponding complete intensity function (Cox and Isham (1985)), where $H_{t}$ is a history of the process up to and including $t$. The useful interpretation is that $\lambda_{c}\left(t, H_{t}\right) d t+o(d t)$ defines the probability of a shock in $(t, t+d t]$ given the history $H_{t}$ (the configuration of shocks in $(0, t]$ ). The complete intensity function is an alternative (to the hazard rate process $\lambda_{t}$ ) way of defining the point process. The conditional hazard can be defined in this case by the following probability:

$$
P\left\{T \in(t, t+d t] \mid H_{t}, T>t\right\}=\theta(t) \lambda_{c}\left(t, H_{t}\right) d t[1+o(1)] .
$$

Assume now that shocks occur in accordance with the NHPP with intensity $\lambda(t)$. It is clear that in this case (compare with definition (4)):

$$
\lambda_{c}\left(t, H_{t}\right) \equiv \lambda(t)
$$

Then relation (6) reduces formally to

$$
P\left\{T \in(t, t+d t] \mid H_{t}, T>t\right\}=\theta(t) \lambda(t) d t(1+o(1)] \equiv \lambda_{r}(t) d t(1+o(1))
$$

and, finally, the corresponding survival probability is given by

$$
\bar{F}(t)=\exp \left\{-\int_{0}^{t} \theta(u) \lambda(u) d u\right\}=\exp \left\{-\int_{0}^{t} \lambda_{r}(u) d u\right\},
$$

Equation (8) obviously follows from the fact that the conditional hazard (26) does not depend on history. Thus we, in fact have proved in a very simple natural way (and, what is more, allowing various generalizations!) the following result: 
Theorem 1. Under stated assumptions the lifesaving model (4) is equivalent to the shock survival model (6)-(8).

Consider the following lifesaving ratio, which can be also of interest for comparing life expectancies of an object in different environments:

$$
R_{\theta}=\frac{\int_{0}^{\infty} \exp \left\{-\int_{0}^{t} \theta(u) \lambda(u) d u\right\} d t}{\int_{0}^{\infty} \exp \left\{-\int_{0}^{t} \lambda(u) d u\right\} d t} .
$$

If, for example $\lambda(t) \equiv \lambda$ and $\theta(t) \equiv \theta$, then $R_{\theta}=\theta^{-1}$.

It is more important for demographic practice to define the lifesaving ratio for the mean remaining lifetime (life expectancy at time $t_{r}$ ), because improvement in medical, socio-economical and environmental conditions usually have a more substantial effect on older people. Relation (9) is modified for this case to

$$
R_{\theta}\left(t_{r}\right)=\frac{\int_{0}^{\infty} \exp \left\{-\int_{t_{r}}^{t_{r}+t} \theta(u) \lambda(u) d u\right\} d t}{\int_{0}^{\infty} \exp \left\{-\int_{t_{r}}^{t_{r}+t} \lambda(u) d u\right\} d t} .
$$

It is clear that under the assumption that $\theta(t) \lambda(t)$ is an increasing (non-decreasing function, $R_{\theta}\left(t_{r}\right)\left(R_{\theta}(0) \equiv R_{\theta}\right)$ should also increase in $t_{r}$. In continuation of the simplest example above, assume that the mortality rate is a step function (young people do not dye!):

$$
\lambda(t)= \begin{cases}0, & 0 \leq t<t_{0} \\ \lambda, & t \geq t_{0}\end{cases}
$$

Then for $0<t_{r}<t_{0}$ :

$$
R_{\theta}\left(t_{r}\right)=\frac{\lambda\left(t_{0}-t_{r}\right)+\theta^{-1}}{\lambda\left(t_{0}-t_{r}\right)+1}<\theta^{-1},
$$

as $\theta^{-1}>1$, and it can be easily seen that $R_{\theta}\left(t_{r}\right)$ is increasing in $t_{r}$.

\section{LIFESAVING IN HETEROGENEOUS POPULATIONS}

The foregoing results were obtained under the assumption of the homogeneous population with mortality rate $\lambda(t)$. Assume now that $F(t)$ is indexed by a random variable $Z: P(T \leq t \mid Z=z) \equiv P(T \leq t \mid z)=F(t, z)$ and that the corresponding probability density function $f(t, z)$ exists. Then the mortality rate $\lambda(t, z)$ is defined, as usually, as $f(t, z) / \bar{F}(t, z)$. Let $Z$ be interpreted as a non-negative random variable with support in $[a, b], a \geq 0, b \leq \infty$ and the density $\pi(z)$. Another meaningful interpretation defines unobserved $Z$ as frailty in heterogeneous population. For the specific multiplicative form of the failure rate the frailty model was introduced and applied to demographic studies by Vaupel et al (1979). The above setting leads naturally 
to considering mixtures of distributions, which are useful for describing heterogeneity. The mixture Cdf is defined in a standard way

$$
F_{m}(t)=\int_{a}^{b} F(t, z) \pi(z) d z,
$$

whereas the mixture failure rate in accordance with the definition (e.g., in Finkelstein and Esaulova (2001)) is

$$
\lambda_{m}(t)=\frac{\int_{a}^{b} f(t, z) \pi(z) d z}{\int_{a}^{b} \bar{F}(t, z) \pi(z) d z}=\int_{a}^{b} \lambda(t, z) \pi(z \mid t) d z,
$$

where the conditional pdf (on condition that $T>t$ )

$$
\pi(z \mid t) \equiv \pi(z \mid T>t)=\pi(z) \frac{\bar{F}(t, z)}{\int_{a}^{b} \bar{F}(t, z) \pi(z) d z} .
$$

It is clear, that the corresponding hazard (mortality) rate process for the heterogeneous case is defined by (compare with (3))

$$
\lambda_{t}=\lambda(t, Z), t \geq 0 \text {. }
$$

On the other hand, the complete intensity function is $\lambda_{c}\left(t, H_{t}\right) \equiv \lambda(t, z)$, whereas equation (7) for $Z=z$ turns to

$$
P\left\{T \in(t, t+d t] \mid H_{t}, T>t\right\}=\theta(t) \lambda(t, z) d t[1+o(1)]
$$

and exponential representation (8) for $Z=z$ can be written as

$$
\bar{F}_{\theta}(t, z)=\exp \left\{-\int_{0}^{t} \theta(u) \lambda(u, z) d u\right\} .
$$

Finally, the survival probability is defined by the following expectation with respect to the random variable $Z$ :

$$
\bar{F}_{m, \theta}(t)=E\left[\exp \left\{-\int_{0}^{t} \theta(u) \lambda(u, Z) d u\right\},\right.
$$

where subscript " $m$ ", as previously, stands for "mixture". The observed mortality rate in accordance with the general definition (Yashin and Manton (1997)), can be written for this case in the following way:

$$
\lambda_{m, \theta}(t)=\theta(t) E[\lambda(t, Z) \mid T>t]=\theta(t) \int_{a}^{b} \lambda(t, z) \pi(z \mid t) d z
$$

The lifesaving ratio for $Z=z$ is defined similar to equation (9):

$$
R_{\theta, z}=\frac{\int_{0}^{\infty} \exp \left\{-\int_{0}^{t} \theta(u) \lambda(u, z) d u\right\} d t}{\int_{0}^{\infty} \exp \left\{-\int_{0}^{t} \lambda(u, z) d u\right\} d t} .
$$

As $R_{\theta, z}$ is not a conditional characteristic, the lifesaving ratio for the heterogeneous population is just an 'ordinary expectation':

$$
R_{E, \theta}=\int_{a}^{b} R_{\theta, z} \pi(z) d z
$$


Similar to the homogeneous case the lifesaving ratio $R_{E, \theta}\left(t_{r}\right)$ can be defined for the remaining lifetime. It follows from definition (18) and properties of $R_{\theta}\left(t_{r}\right)$, that $R_{E, \theta}\left(t_{r}\right)$ also increases in $t_{r}$.

The model can be easily generalized to the case when probability of lifesaving (or of failure in reliability setting) also depends on $Z$ :

$$
\begin{gathered}
\bar{F}_{m, \theta}(t)=E\left[\exp \left\{-\int_{0}^{t} \theta(u, Z) \lambda(u, Z) d u\right\},\right. \\
\lambda_{m, \theta}(t)=E[\theta(t, Z) \lambda(t, Z) \mid T>t]=\int_{a}^{b} \theta(t, z) \lambda(t, z) \pi(z \mid t) d z
\end{gathered}
$$

Let the values of $\lambda(t, z)$ be ordered with respect to $z$ :

$$
\lambda\left(t, z_{1}\right)<\lambda\left(t, z_{2}\right), \quad z_{1}<z_{2}, \forall z_{1}, z_{2} \in[a, b], t \geq 0
$$

For ordered values of $\lambda(t, z)$ it is also reasonable to suppose that $\theta(t, z)$ is also increasing in $z$ : individuals with larger values of the mortality rate are likely to be less resistant to shocks as well. Define the following unconditional expectation to be compared with conditional expectation (20):

$$
\lambda_{P, \theta}(t)=\int_{a}^{b} \theta(t, z) \lambda(t, z) \pi(z) d z
$$

The following result, defining the simple lower bound for the survival probability, is rather straightforward:

Theorem 2. Let $\lambda(t, z)$ and $\theta(t, z)$ be ordered in $z$. Then

$$
\lambda_{m, \theta}(t)<\lambda_{P, \theta}(t), t>0
$$

and

$$
\bar{F}_{m, \theta}(t)=\exp \left\{-\int_{a}^{b} \lambda_{m, \theta}(u) d u\right\}>\exp \left\{-\int_{a}^{b} \lambda_{P, \theta}(u) d u\right\}
$$

Proof. It is clear that under given assumptions the reduced mortality rate $\theta(t, z) \lambda(t, z)$ is also ordered in $z$. This means that the principle: "the weakest populations are dying out first", which describes the bending down property of the observed mortality rate in frailty models, can be applied to the family of mortality rates $\theta(t, z) \lambda(t, z) ; z \in[a, b]$, (Finkelstein and Esaulova (2001)). Inequalities (23) and (24) then follow immediately.

\section{DISCUSSION}

In this section we will discuss some of the obtained results and describe several topics for future studies.

1. The possible generalization of models (2) and (3) is the general repair process (Kijima (1989), Finkelstein (2000)), when the age of an object after the repair at time $t$ is intermediate: $0 \leq x^{\prime} \leq t \quad x^{\prime} \geq 0$. Specifically, $x^{\prime}=0$ defines the perfect repair and $x^{\prime}=t$ defines the minimal repair. Thus, the remaining lifetime survival function after the failure, which occurred at $t$ is defined by (compare with (1)):

$$
\bar{F}\left(t \mid x^{\prime}\right)=\frac{\bar{F}\left(t+x^{\prime}\right)}{\bar{F}\left(x^{\prime}\right)} \text {. }
$$


Applying operation (25) to each repair, leads to the following survival function of the $n$th cycle:

$$
\bar{F}_{n}(t) \equiv \bar{F}\left(t \mid X_{n-1}=x^{\prime}\right)=\frac{\bar{F}\left(t+x^{\prime}\right)}{\bar{F}\left(x^{\prime}\right)}, n \geq 2,
$$

where $X_{n-1}$ is the virtual age of an object immediately after the $n-1$ th repair. The notion of virtual age is well defined for Cdfs with monotone (usually increasing) failure rate $\lambda(t)$ (Kijima (1989), Finkelstein (2000)). On the first cycle $\left[0, t_{1}\right]$ the virtual age is equal to the chronological age. After the first repair, the age of our object is, for instance, reduced to some value $x_{1} \leq t_{1}$, which is the initial virtual age for the second cycle etc. Thus, along with the repair process $T_{n}, n \geq 1$, the virtual age process $X_{n-1}, n \geq 2$ can be also defined. The corresponding hazard rate process can be defined as

$$
\lambda_{t}=\sum_{n=0}^{\infty} \lambda\left(t-T_{n}+X_{n}\right) I\left(T_{n}<t \leq T_{n+1}\right), T_{0}=0, X_{0}=0
$$

It can be easily seen that for $X_{n}=0, n \geq 1$ this process reduces to the renewal model (2) and for $X_{n}=T_{n}, n \geq 1$ it defines the NHPP (3). The next step is to specify the model. The most natural assumption is the linear model:

$$
X_{n}=q\left(X_{n-1}+T_{n}\right), n=1,2, \ldots ; X_{0}=0
$$

where $q>0$. Thus the repair action proportionally reduces $(q<1)$ the value of the virtual age which an object had just before the current failure.

2. Combining the general repair model with the lifesaving procedure of the previous section can be an interesting problem for the future research, though it seems to be very complicated from the technical point of view. It is worth noting that even for the specific case of the renewal process (perfect repair) one cannot solve this problem easily, as for the case of minimal repair and bounds for the survival probability are usually obtained (Finklstein (2003)). Therefore, the minimal repair assumption for the homogeneous and heterogeneous cases is very important for the current study.

3. It is interesting to compare the impacts of a single shock on homogeneous and heterogeneous populations. Let the mortality rate in the absence of a shock for the homogeneous population be $\lambda(t)$. A shock, occurring at $t=x$, results in death (failure) with probability $\theta(x)$. This means that the resulting failure rate can be described by the $\delta$-function at $t=x$ (the jump $\theta(x)$ at this instant of time in the corresponding discrete -continuous Cdf).

Assume that $\lambda(t, z)$ and $\theta(t, z)$ for the heterogeneous case are ordered as in Section 3 . Then the resulting failure rate can be also described by the $\delta$-function at $t=x$. The jump at this instant of time in the corresponding Cdf is

$$
\theta_{m}(x)=\int_{a}^{b} \theta(t, z) \pi(z \mid x) d z .
$$

It is clear and can be easily proved analytically that after the shock the resulting failure rate in $(x, \infty)$ will be smaller than $\lambda_{m}(t)$ defined by relation $(11)$, as the weaker populations are affected by the shock to the larger extent.

4. The proportional hazard-type model (4) can be used for modeling an impact of environment on the lifetime characteristics of an object. The function $\theta(t)$ in this case does not have the meaning of probability, whereas $\lambda(t)$ can be interpreted as 
some baseline mortality rate Coming back to a single shock setting of the previous comment, we can consider a non-instantaneous shock, described by the function $\theta(t)$ in (4). Assume that this function is equal to 1 in $[0, x-\sigma]$ and in $(x+\sigma, \infty)$, increases in $(x-\sigma, x]$ to the value $\theta(x)>1$ and then decreases to 1 in $(x, x+\sigma]$ (for some relatively small $\sigma>0)$. The resulting mortality rate for the homogeneous population is trivially defined by equation (4), whereas for the heterogeneous population (with ordered for simplicity $\lambda(t, z), \theta(t, z)$ and under some other assumptions to be defined) it is equal to $\lambda_{m}(t)$ in $[0, x-\sigma]$; exceeds $\lambda_{m}(t)$ in $\left(x-\sigma, x+\sigma^{\prime}\right]$, where $\left|\sigma^{\prime}\right|<\sigma$ can be obtained analytically, and finally, it is smaller than $\lambda_{m}(t)$ in $\left(x+\sigma^{\prime}, \infty\right)$. The described shape is similar to the shape of the force of mortality in heterogeneous population affected by a shock, which was analyzed in Vaupel (2002) in a demographic application.

\section{References}

Aven T., and Jensen U. (1999) Stochastic Models in Reliability. Springer.

Aven T., and Jensen U. (2000). A general minimal repair model. J. Appl. Prob., 37, 187-197.

Barlow R., and Proschan F. (1975). Statistical Theory of Reliability and Life Testing. Probability Models, Holt, Rinehart and Winston, New-York

Bergman B. (1985). On reliability theory and its applications. Scand. J. Statistics, 12, $1-41$.

Block, H.W., Savits T., and Borges W. (1985). Age dependent minimal repair. $J$. Appl. Prob., vol 22, pp.370-386.

Boland P.J., and El-Neweihi E. (1998). Statistical and information based minimal repair for k out of n systems. J. Appl. Prob., 35, 731-740.

Brown M., Proschan F. (1983). Imperfect repair. J. Appl. Prob., 20, 851-862.

Cox, D.R. and Isham, V. (1980). Point Processes, New York: Chapman and Hall.

Finkelstein, M.S. (1992). Some notes on two types of minimal repair. Adv. Appl. Prob. 24, 226-228.

Finkelstein M. S (1997). The concealed age of distribution functions and the problem of general repair. J. Statist. Plann. Inference, 1997, 65, 315-321.

Finkelstein M.S. Wearing-out components in variable environment (1999). Reliability Engineering and System Safety, 66, N3, 235-242.

Finkelstein M.S. (2000). Modeling a process of non-ideal repair. In: Recent Advances in Reliability Theory. Limnios N., Nikulin M. (eds). Birkhauser, 41-53.

Finkelstein, M.S., and V. Esaulova, V (2001) Modeling a failure rate for the mixture of distribution functions Probability in Engineering and Informational Sciences, 15, 383-400.

Finkelstein M.S. Simple bounds for terminating Poisson and renewal processes. Journal of Statistical Planning and Inference, 113, 541-548

Kijima M. Some results for repairable systems with general repair. J. Appl. Prob., 1989, 26, 89-102.

Natvig B. (1990). On information-based minimal repair and reduction in remaining system lifetime due to a failure of a specific module. J. Appl. Prob., 27, 365-375.

Nelson W. Accelerated Testing. (1990) John Wiley \& Sons, New York.

Vaupel, J.W., and Yashin, A.I. (1987). Repeated resuscitation: how life saving alters life tables. Demography, 4, 123-135 
Vaupel J.W., Manton K.G., and Stallard E. (1979). The impact of heterogeneity in individual frailty on the dynamics of mortality. Demography,16, 439-454.

Vaupel J.W. (2002). Personal communication.

Yashin A.I., and Manton K.G. (1997) Effects of unobserved and partially observed covariate processes on system failure: a review of models and estimation strategies. Statistical Science, vol. 12, pp. 20-34.

Yashin, A.I., Iachin, I., and Begun, A.S. (2000). Mortality modeling: a review. Mathematical Population Studies, 8, 305-332. 